\title{
Ouabain induces the extinction of contextual fear memory in rats subjected to chronic unpredictable stress.
}

\author{
Cristoforo Scavone (GUEST EDITOR $)^{1}$, Jacqueline Leite ${ }^{1}$, Ana Orellana ${ }^{1}$, Amanda \\ Mamutomo ${ }^{1}$, Diana Andreotti ${ }^{1}$, Larissa Lima ${ }^{1}$, Vinicius Nakao ${ }^{1}$, Elisa Kawamoto ${ }^{1}$, and \\ Carolina Munhoz ${ }^{1}$ \\ ${ }^{1}$ University of Sao Paulo
}

November 24, 2020

\begin{abstract}
Ouabain (OUA) is an inhibitor of $\mathrm{Na}+, \mathrm{K}+$-ATPase that has been identified as an endogenous substance present in human plasma, and it has been shown to be associated with the response to acute stress in both animals and humans. Chronic stress is a major aggravating factor of psychiatric disorders, including depression and anxiety. The present work investigates the effects of OUA intermittent administration during chronic unpredictable stress (CUS) protocol in the rat's central nervous system (CNS). Adult male Wistar rats were pretreated intraperitoneally with ouabain $(1.8 \mu \mathrm{g} / \mathrm{kg})$, followed by CUS protocol for 14 days. The levels of serum corticosterone, ACTH, and CRH serum were evaluated through ELISA and the expression of CRH, CRHR1, and CRHR2 genes in the hypothalamus and hippocampus of the animals through RT-PCR. Inflammatory parameters were also investigated, as well as the behavioral CUS effects on memory, that were assayed through the object recognition task, contextual fear conditioning, and memory extinction paradigms. The results suggest that intermittent OUA treatment reversed CUS-induced HPA axis hyperactivity through the reduction of (i) glucocorticoids levels, (ii) CRH-CRHR1 expression, and by decreasing neuroinflammation with the reduction of iNOS activity, without interfering with the expression of antioxidant enzymes. These changes in both the hypothalamus and hippocampus may reflect in the rapid extinction of aversive memory. The present data demonstrate, for the first time, the ability of OUA to modulate the HPA axis as well as the disappearance of aversive memory in rats.
\end{abstract}

\section{Hosted file}

BJPJacqLeite.pdf available at https://authorea.com/users/378262/articles/494811-ouabaininduces-the-extinction-of-contextual-fear-memory-in-rats-subjected-to-chronicunpredictable-stress 
Figure 1A Experimental design of Chronic Unpredictable Stress (CUS) protocol

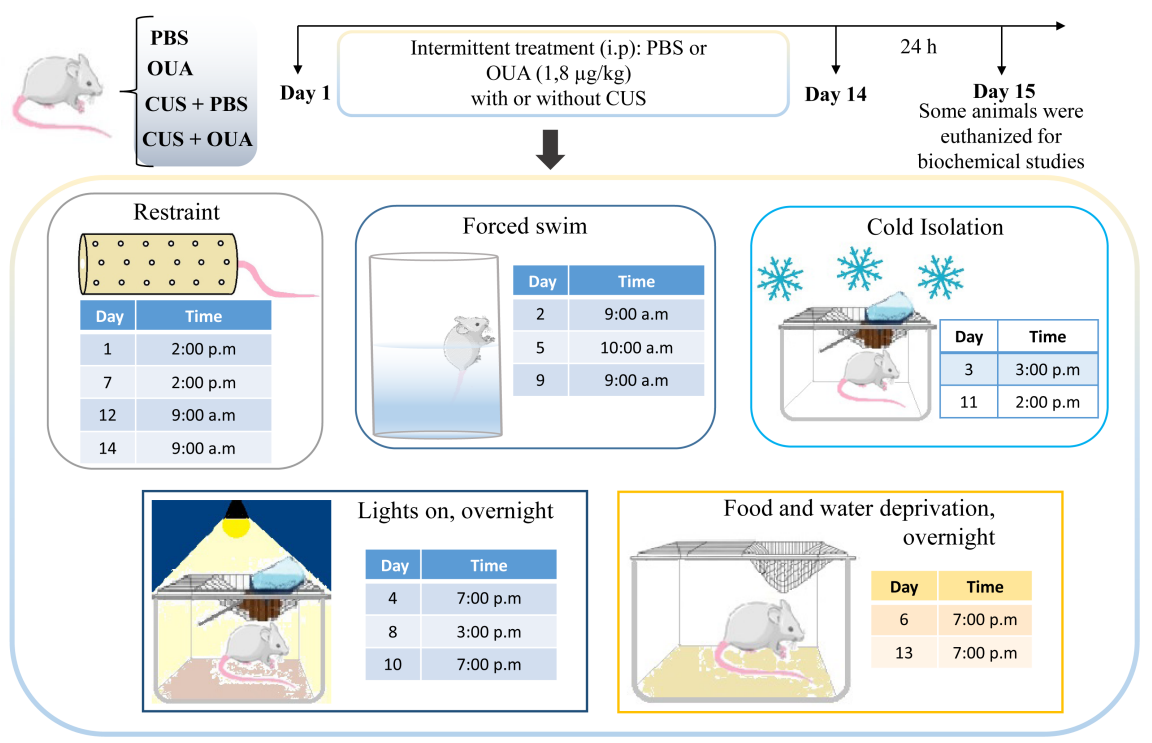

Figure 1B, 1C

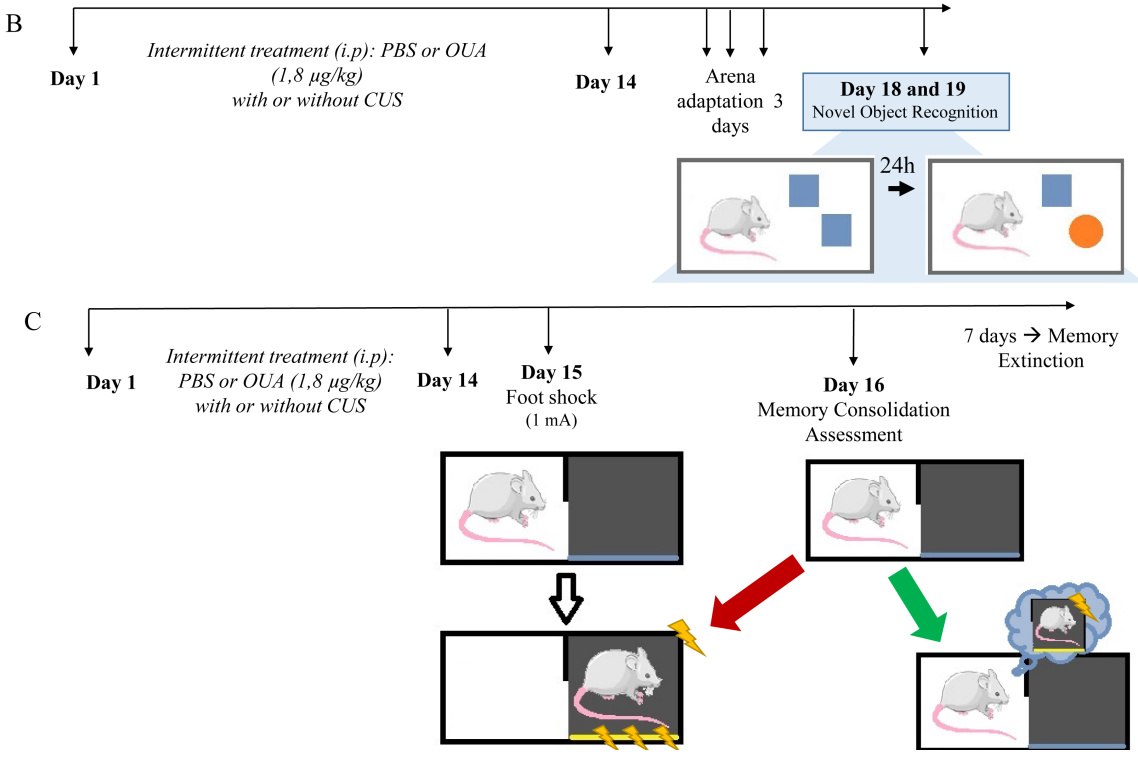


Figure 2
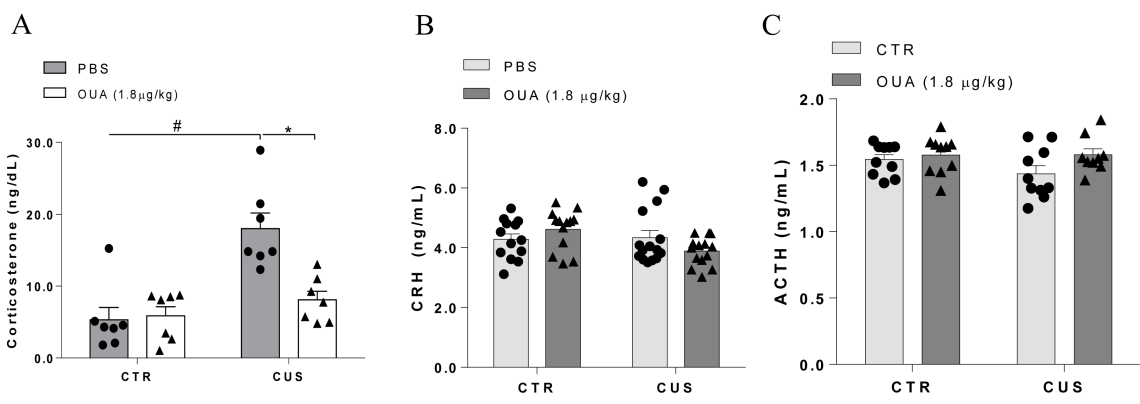

Figure 3
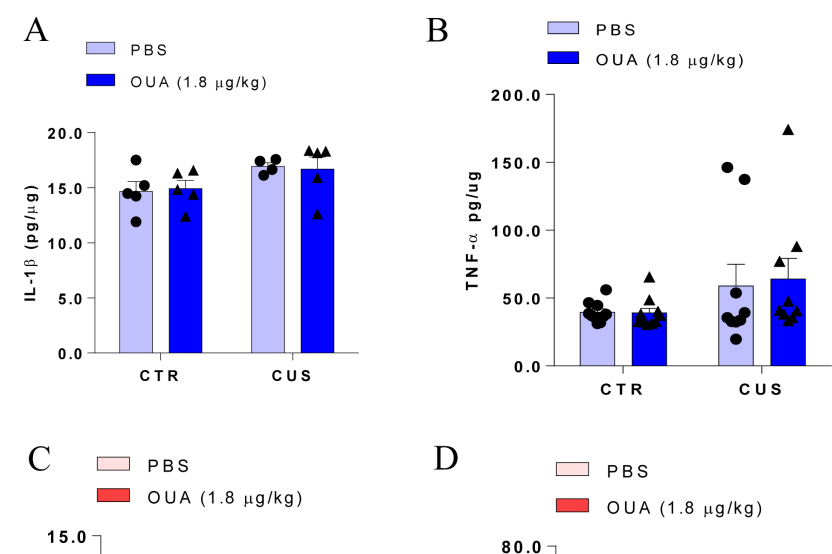

D
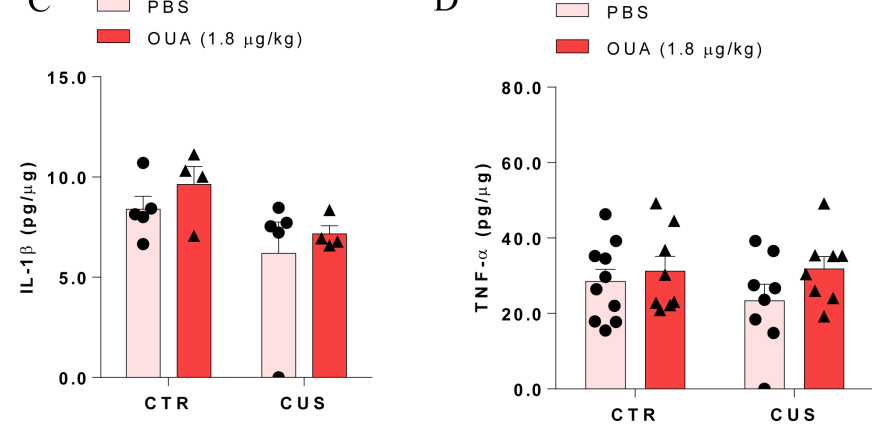
Figure 4
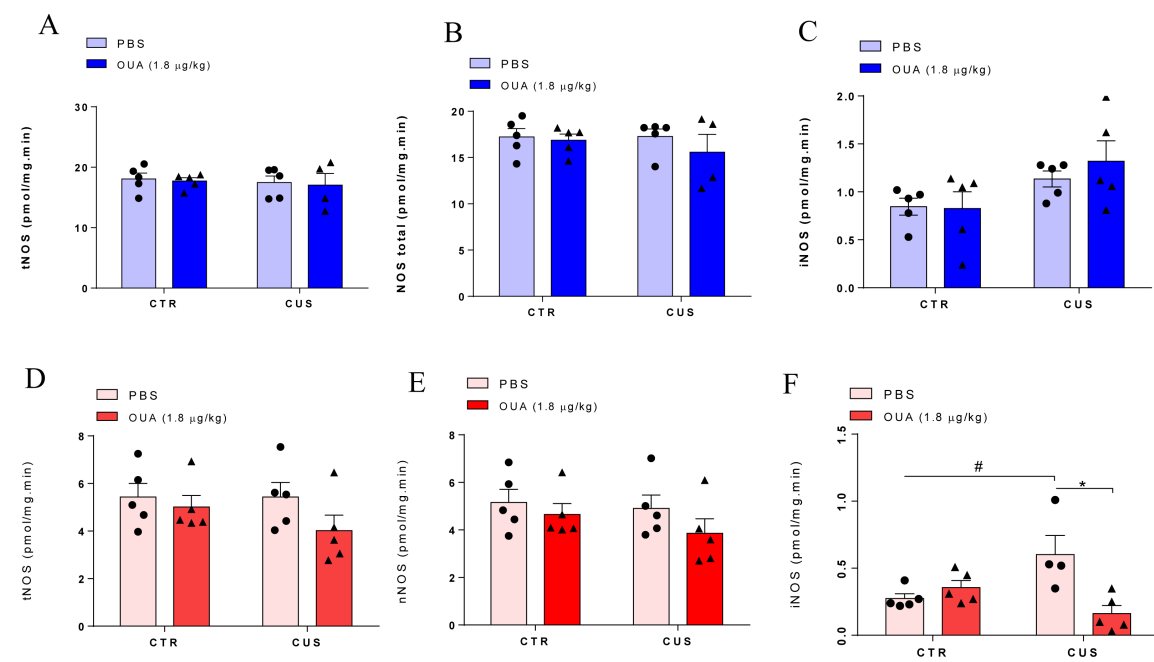

$\mathrm{E} \square$ PBS
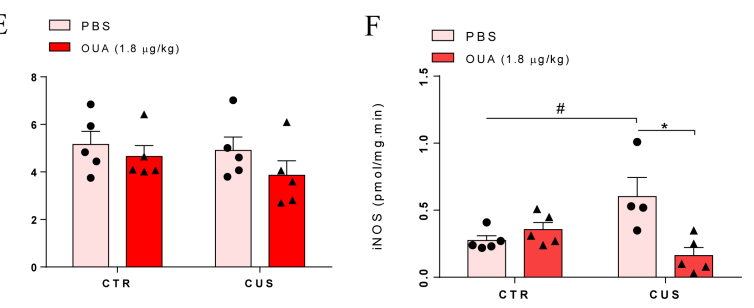

Figure 5
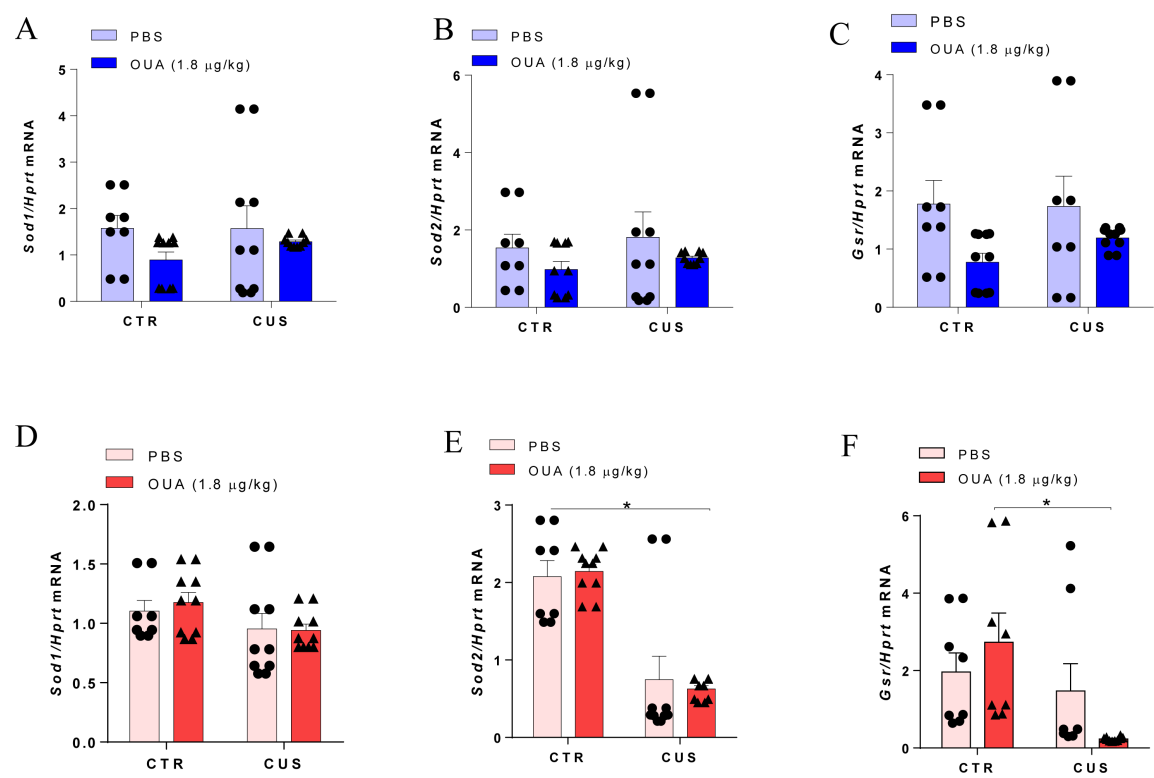
Figure 6

A

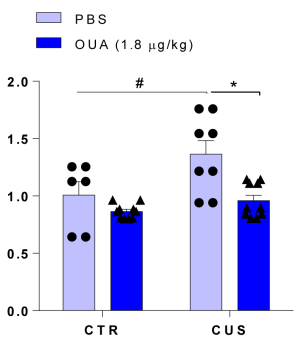

D

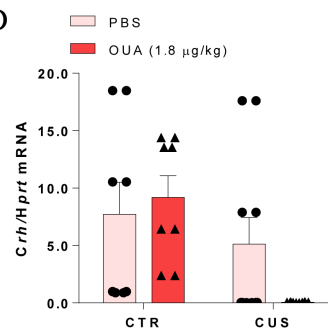

B

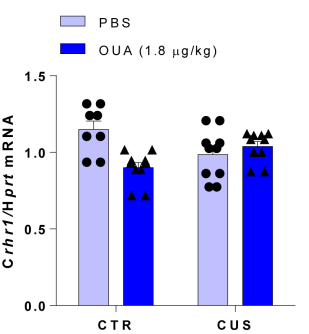

E

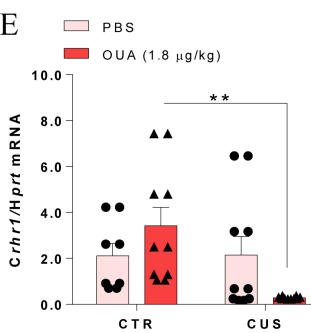

C

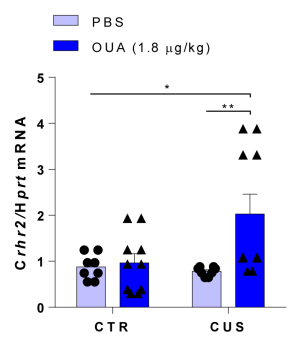

F $\square$ PBS

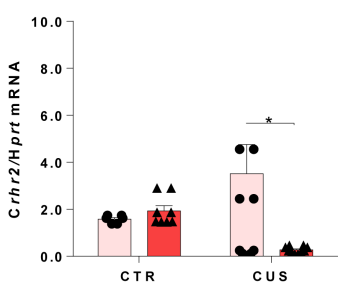

Figure 7

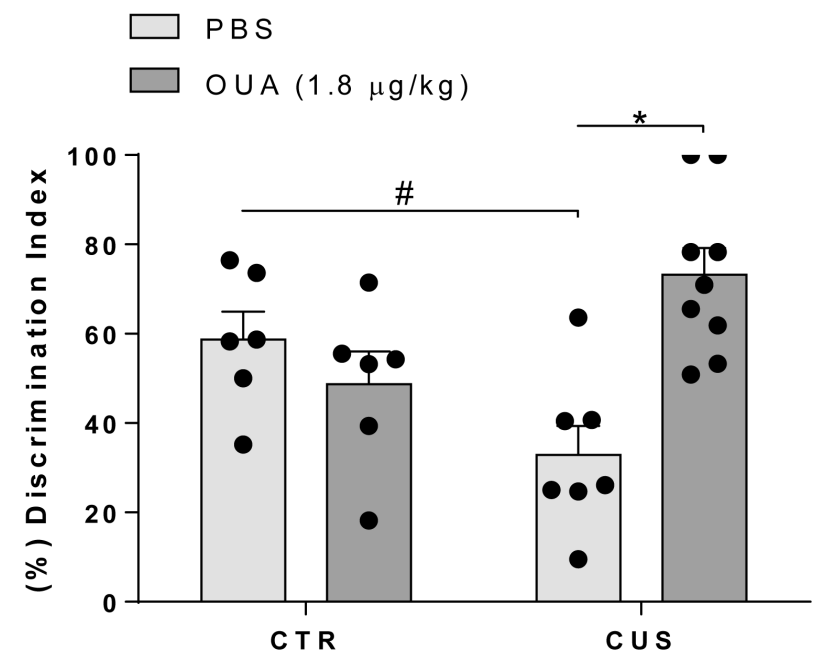


Figure 8
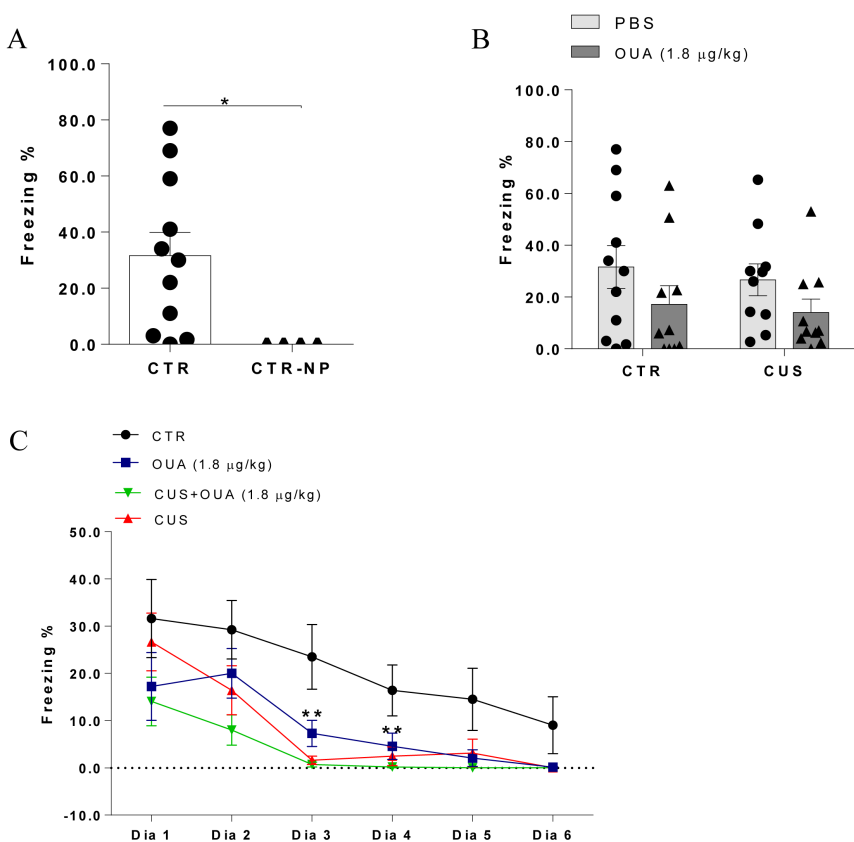

Figure 9

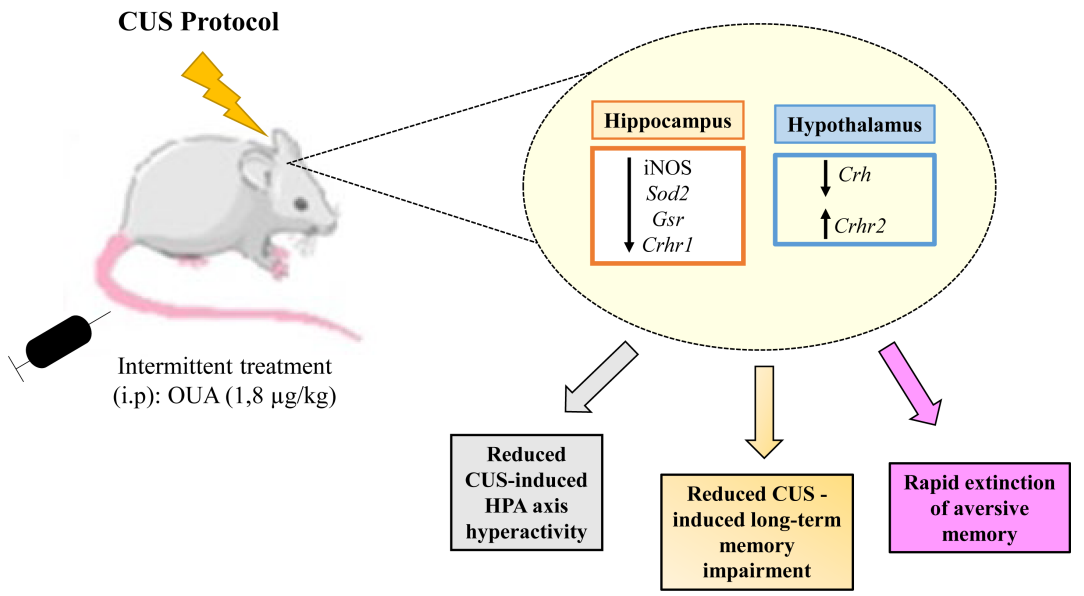

